\title{
notes
}

\section{Le rôle du groupe intersyndical de l'industrie nucléaire dans la radioprotection des travailleurs}

\author{
P. BOIRON, J. CAZAUX (*) \\ (Manuscrit reçu le 31 juillet 1985)
}

\section{INTRODUCTION}

Le Groupe intersyndical de l'industrie nucléaire (GIIN) a été créé en 1959 par douze fédérations professionnelles regroupant des industries impliquées dans les études et réalisations nucléaires. Son objet est :

a) d'étudier et de préciser, à l'usage des groupements professionnels membres, les positions des entreprises industrielles françaises à l'égard des recherches et fabrications d'ordre nucléaire ;

b) de constituer, entre ses membres, l'élément de liaison spécialisé dans les questions qui intéressent particulièrement l'industrie nucléaire ;

c) de représenter auprès des pouvoirs publics, administrations, établissements publics ou privés, organismes professionnels nationaux ou internationaux, les intérêts spécifiques généraux de l'industrie nucléaire française, en vue d'informer, de conseiller et de protéger cette dernière dans toutes les questions se rapportant à son activité particulière.

Très tôt, le GIIN s'est préoccupé de la radioprotection des travailleurs de l'industrie nucléaire dans le souci d'éclairer ses adhérents sur leur responsabilité en la matière et de participer à la codification des pratiques découlant de la réglementation.

C'est dans ce cadre que le GIIN a créé :

- le carnet du travailleur directement affecté à des travaux sous rayonnement (personnel DATR) ;

- l'aide-mémoire de radioprotection ;

- les stages de formation complémentaire des médecins du travail en matière de surveillance du personnel DATR.

\section{LE CARNET DU TRAVAILLEUR DATR}

Le besoin d'un document individuel que chaque travailleur DATR puisse avoir constamment sur lui, sorte de passeport reconnu par l'employeur et le chef de l'établissement classé dans lequel s'effectue la mission, a été perçu dès le début du programme électronucléaire dans le cadre d'échanges de vues entre EdF et les constructeurs.

(*) 15, rue Beaujon, 75008 Paris. 
Un tel document n'était pas visé par la réglementation. II pouvait être renseigné et validé par l'employeur et tenu à jour par le travailleur luimême. Son application a été testée pendant dix mois à Fessenheim, puis étendue à d'autres sites nucléaires, pour être aujourd'hui d'un usage généralisé.

II présente, sous un format réduit, la situation du travailleur vis-à-vis des différentes exigences de la réglementation :

- formation ;

- surveillance médicale ;

- doses reçues durant les différentes périodes de référence.

II remplit aussi son rôle de passeport pour chaque entrée en zone contrôlée en ce qui concerne la situation du travailleur vis-à-vis des exigences de la réglementation s'imposant à l'employeur. Soulignons que ce carnet ne remplace pas la fiche d'irradiation du dossier médical sur laquelle figurent, non pas les lectures quotidiennes du dosimètre individuel, mais les doses cumulées déduites du développement des films qui ne sont connues que plus tard.

EdF utilise le carnet DATR du GIIN pour son propre personnel et l'impose aux entreprises travaillant sur ses sites nucléaires depuis 1979. De l'ordre de 80000 exemplaires en ont été vendus à ce jour.

\section{L'AIDE-MEMOIRE DE RADIOPROTECTION}

L'article 11 du décret du 28 avril 1975 (décret n 75-306) précise que l'employeur doit préparer au travail sous rayonnement tout travailleur affecté dans la zone contrôlée ou appelé à y travailler occasionnellement. Cette préparation implique la formation du travailleur généralement dispensée par des organismes spécialisés (organismes de formation ou services spécialisés des grandes entreprises) et son information par une notice appropriée dont la remise par l'employeur donne lieu à émargement de l'intéressé.

Le carnet Radioprotection du GIIN a pour objet de permettre à l'employeur de satisfaire à cette dernière demande. II rappelle des notions de base en matière de radioprotection et, dans sa partie pratique, il est conforme aux prescriptions des principaux exploitants des installations nucléaires de base (EdF, CEA, COGEMA). L'exploitant d'une installation nucléaire de base (INB) est, en effet, responsable au titre de l'article 2 du décret susvisé, de toutes les "mesures générales d'ordre administratif et technique, notamment en matière d'organisation du travail, nécessaires pour la prévention des accidents et des maladies professionnelles susceptibles d'être causés par les rayonnements ionisants".

Pour y satisfaire, ainsi qu'à sa propre responsabilité d'employeur, EdF édite également un carnet de prescriptions destiné au personnel appelé à intervenir en centrales nucléaires. La cohérence des deux ouvrages a été vérifiée. Le GIIN assure leur diffusion aux entreprises adhérentes à ses fédérations membres. 


\section{STAGES DE FORMATION COMPLEMENTAIRE DES MEDECINS DU TRAVAIL}

Les recommandations formulées dans un cadre international se sont traduites par l'exigence réglementaire d'une surveillance médicale spéciale des travailleurs DATR. Cette surveillance est du ressort du médecin du travail. Dans les différentes situations qu'il est appelé à rencontrer, le médecin du travail doit formuler des avis médicaux de valeur. II lui faut pouvoir acquérir, souvent en peu de temps, un complément de connaissances théoriques et pratiques pour bien apprécier les situations et fonder ses diagnostics ou ses recommandations.

Pour satisfaire à un tel besoin et afin de permettre que le dialogue entre le médecin du travail, l'employeur et les travailleurs DATR soit aussi satisfaisant que possible, le GIIN organise annuellement, sous l'égide de la Société française de radioprotection (SFRP), un stage de formation complémentaire en radioprotection réservé à des médecins du travail. Ce stage leur apporte les rappels nécessaires et le complément d'information pratique sur les travaux en milieu radioactif, l'organisation et les moyens de surveillance, leur permettant d'intervenir avec assurance dans leurs différentes missions et en particulier pour aider à la clarté du dialogue entre l'employeur et les travailleurs DATR. 